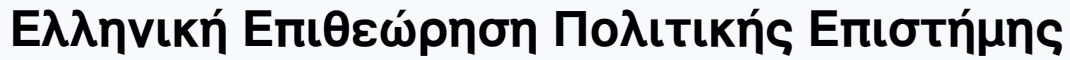

Tóp. 34 (2009)

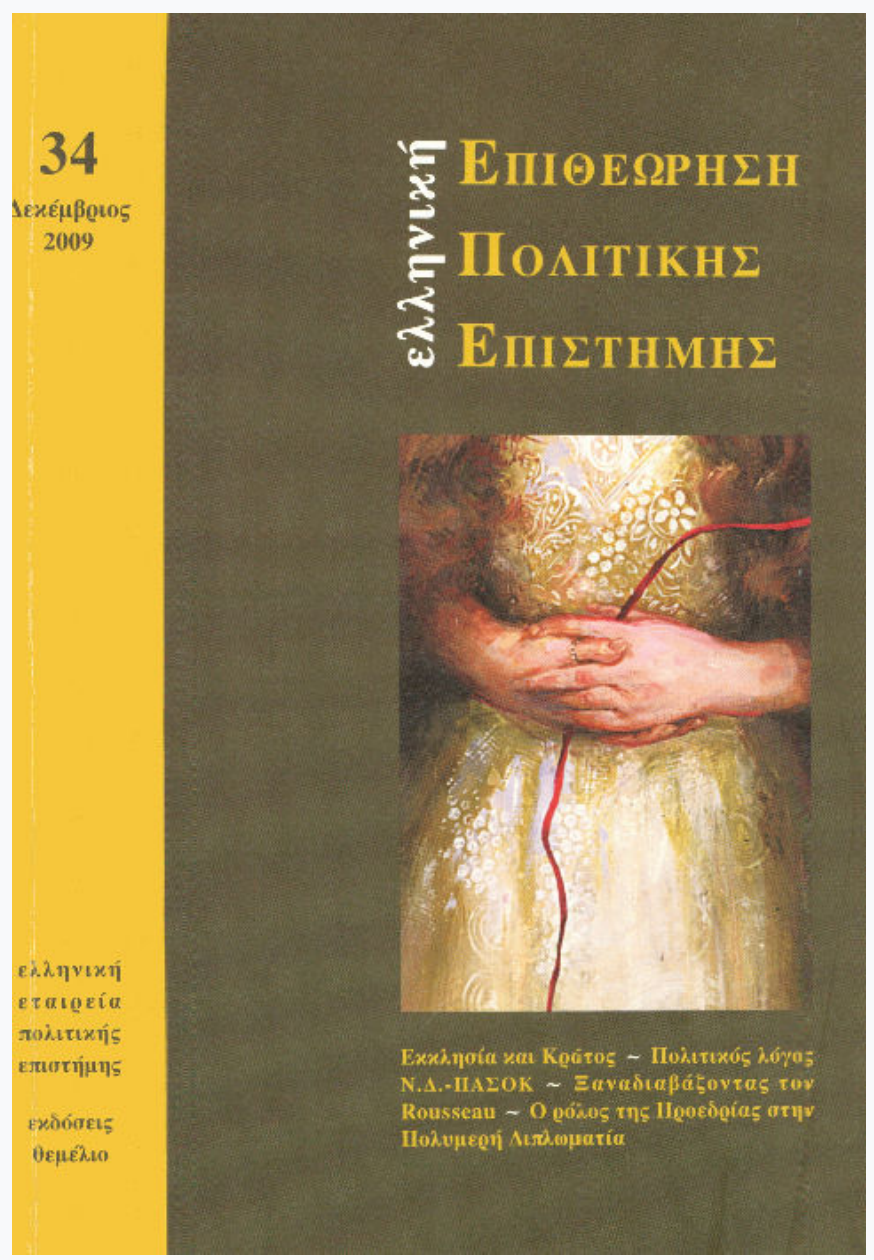

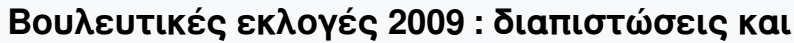 aropís}

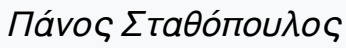

doi: $10.12681 / \mathrm{hpsa} .14488$

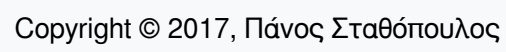

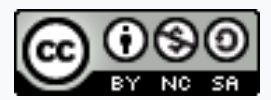

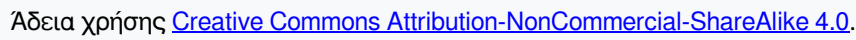

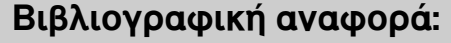

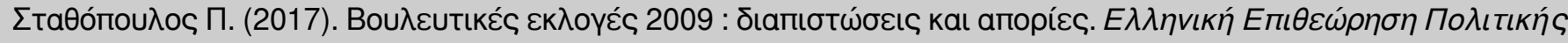
Епıбти́ 


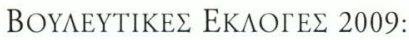

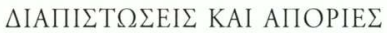

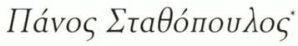

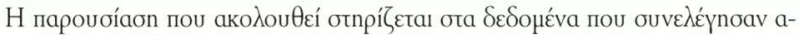

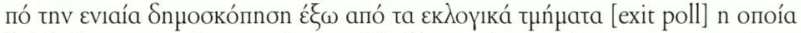

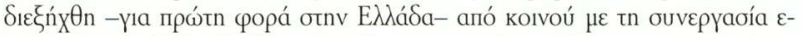

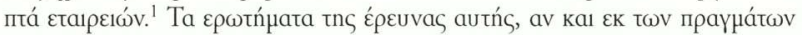

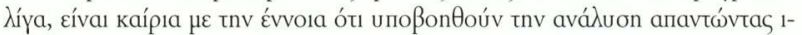

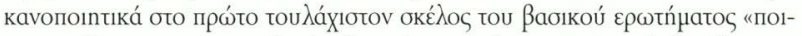

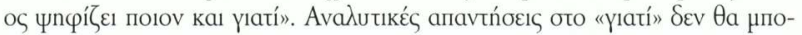

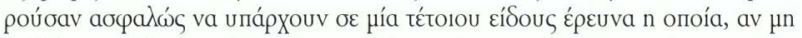

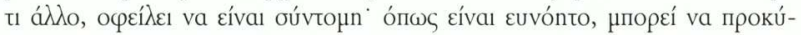

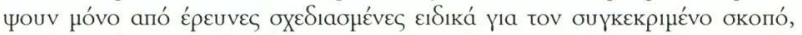

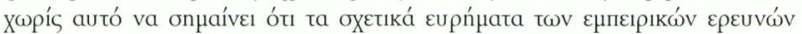

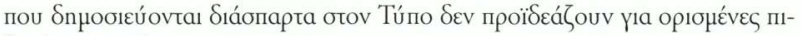

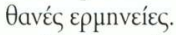

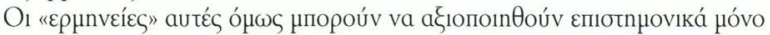

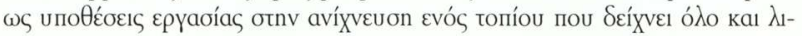

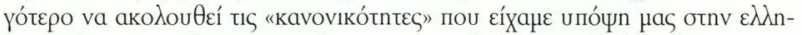

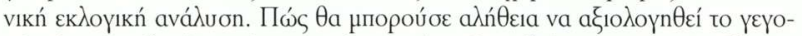
vós ótı ous ßou

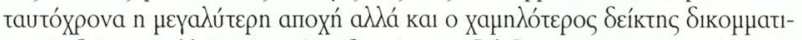

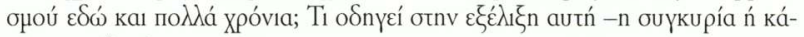

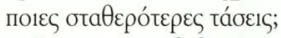

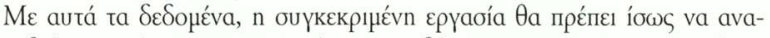

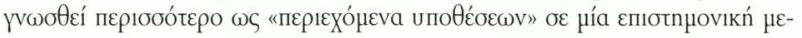

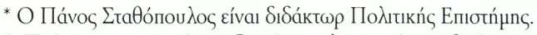

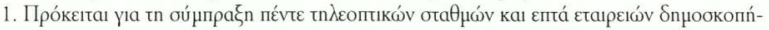

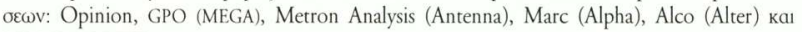
MRB, RASS (NET). 


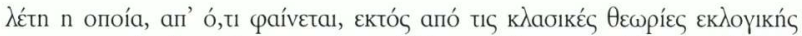

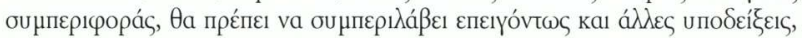

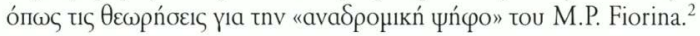

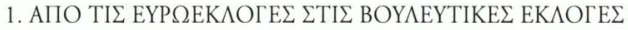

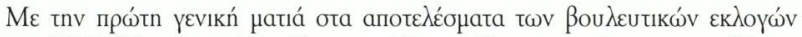

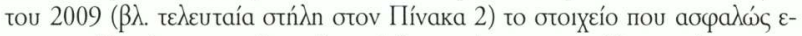

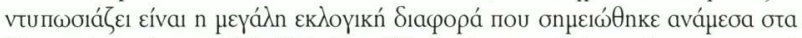

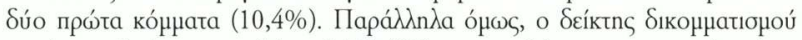

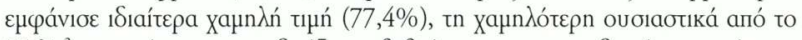

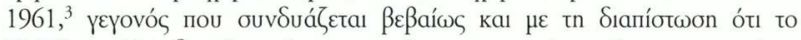

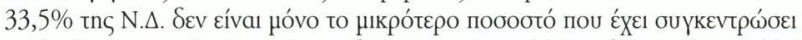

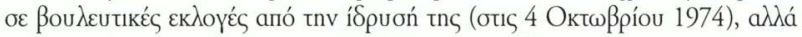

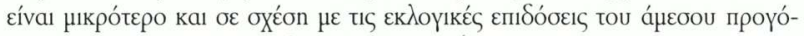

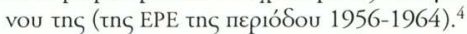

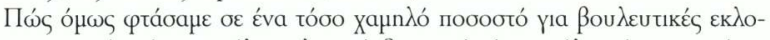

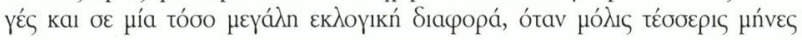

2. M.P. Fiorina, Retrospective Voting in American National Elections, Yale University Press,

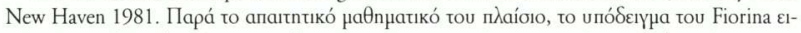

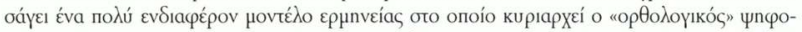

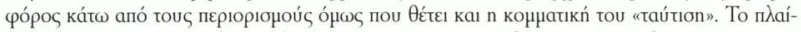

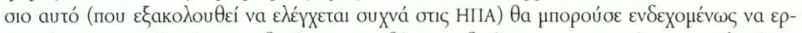

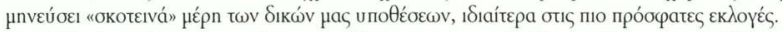

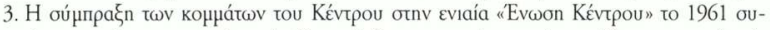

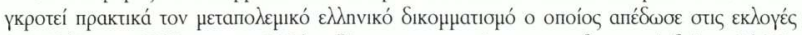

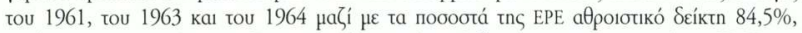

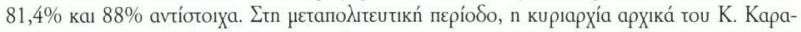

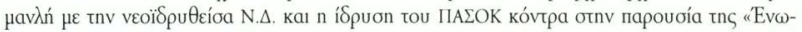

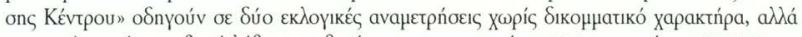

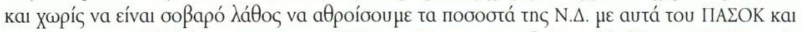

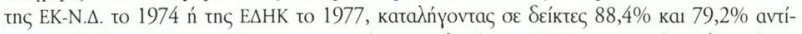

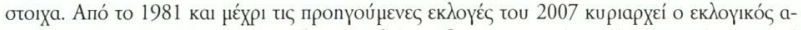

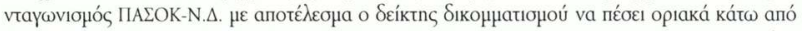

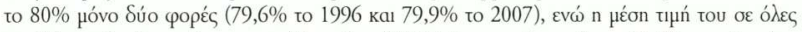

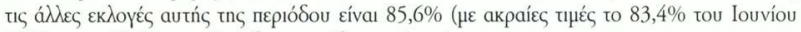

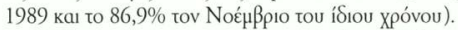

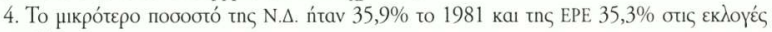
tou 1964. 


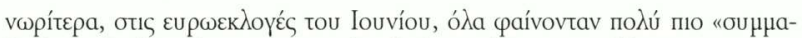

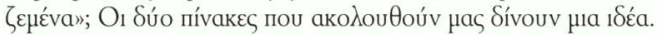

\section{MINAKA 1}

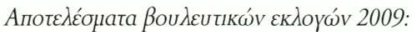

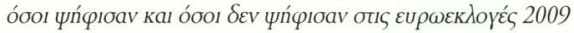

\begin{tabular}{|c|c|c|c|}
\hline & \multirow{2}{*}{ 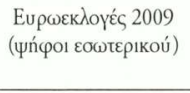 } & \multicolumn{2}{|c|}{ Bou } \\
\hline & & 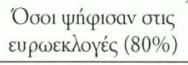 & 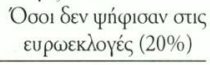 \\
\hline ПАЕОК & 36,7 & 44,9 & 39,9 \\
\hline N. $\Delta$. & 32,3 & 34,7 & 28,5 \\
\hline KKE & 8,3 & 7,4 & 8,2 \\
\hline$\triangle A O \Sigma$ & 7,1 & 5,2 & 7,1 \\
\hline EYPIZA & 4,7 & 4,0 & 6,8 \\
\hline Oıкоһóүo1-Пра́oıvo1 & 3,5 & 1,8 & 5,4 \\
\hline 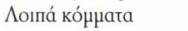 & 7,4 & 2,0 & 4,1 \\
\hline 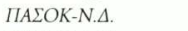 & 4,4 & 10,2 & 11,4 \\
\hline ПАЕОK+N.A. & 69,0 & 79,6 & 68,4 \\
\hline
\end{tabular}

\section{MINAKA 2}

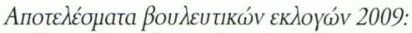

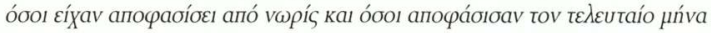

\begin{tabular}{|c|c|c|c|}
\hline & Апочабібце́vоІ $(74,6 \%)$ & Avanоча́бıбто (25,4\%) & ¿úvoגo \\
\hline ПАЕОК & 46,9 & 35,2 & 43,9 \\
\hline N. $\Delta$. & 35,5 & 27,6 & 33,5 \\
\hline KKE & 7,9 & 6,4 & 7,5 \\
\hline$\triangle A O E$ & 3,9 & 10,4 & 5,6 \\
\hline ¿YPIZA & 3,4 & 8,0 & 4,6 \\
\hline Oıкоһóүoı-Пра́ơvo1 & 1,0 & 6,7 & 2,5 \\
\hline ヘопп́ & 1,3 & 5,6 & 2,3 \\
\hline ПАЕОK-N.A. & 11,4 & 7,6 & 10,4 \\
\hline ПIALOK+N.A. & 82,4 & 62,8 & 77,4 \\
\hline
\end{tabular}

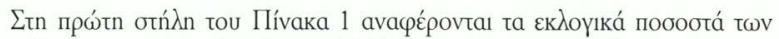

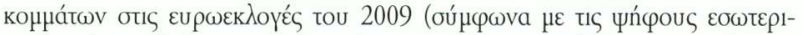

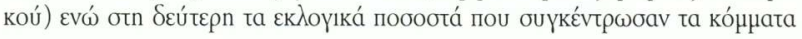




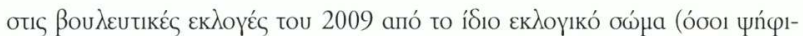

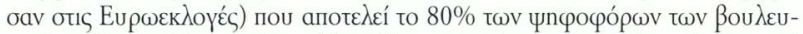

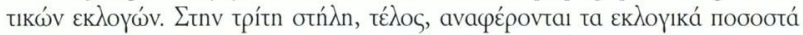

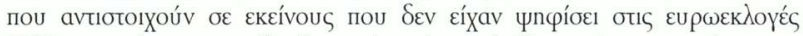

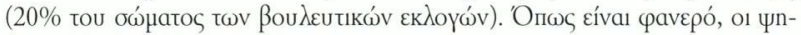

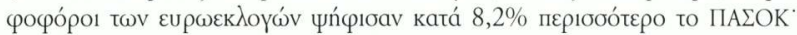

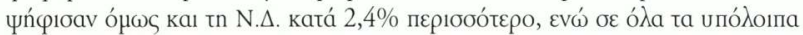

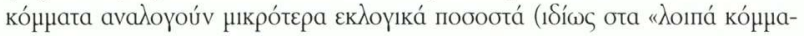

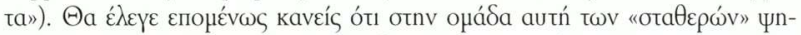

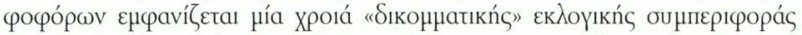

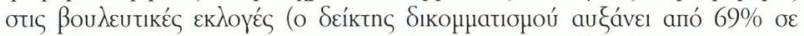

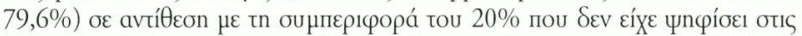

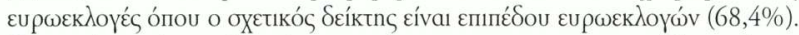

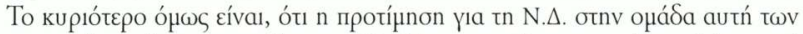

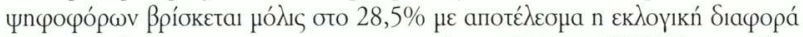

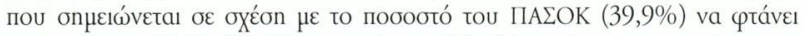

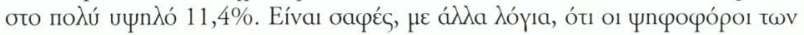

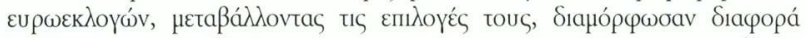

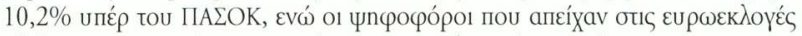

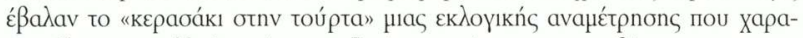

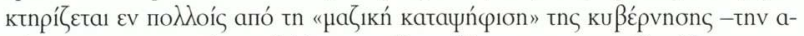

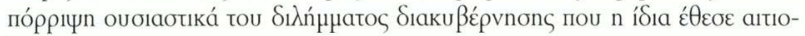

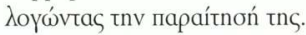

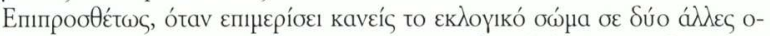

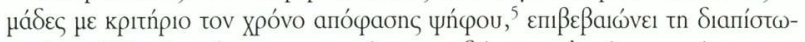

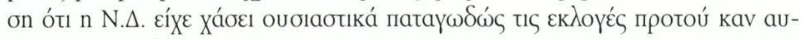

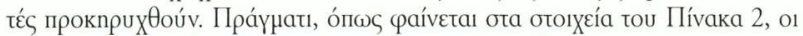

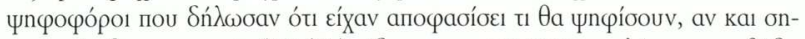

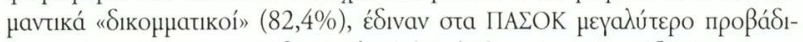

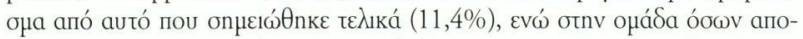

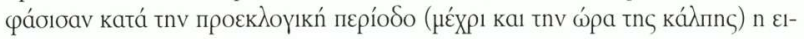

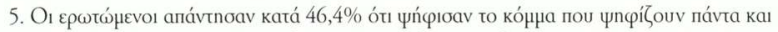

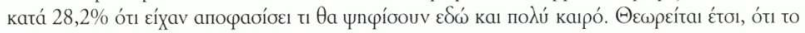

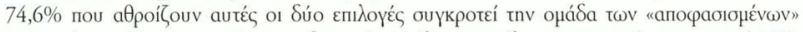

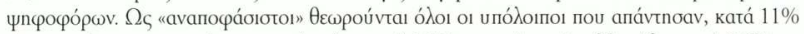

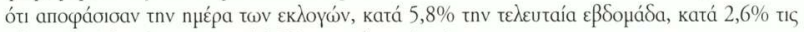

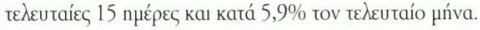




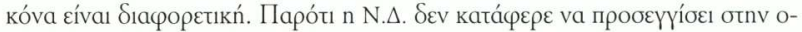

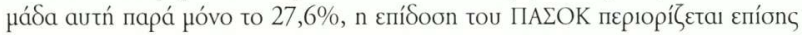

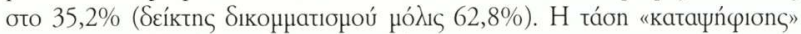

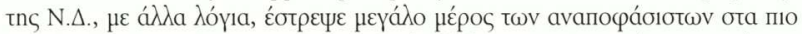

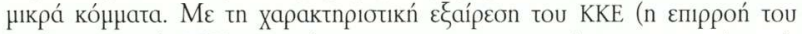

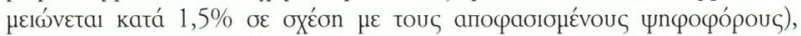

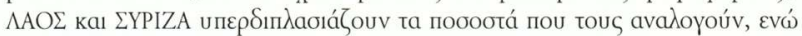

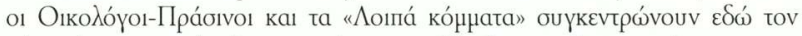

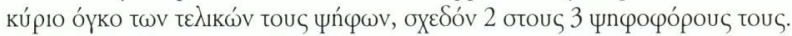

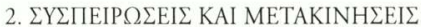

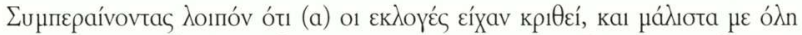

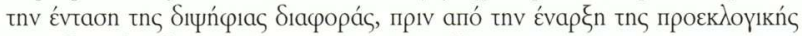

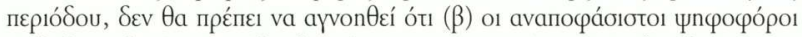

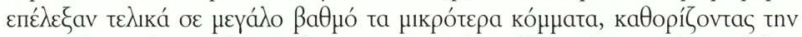

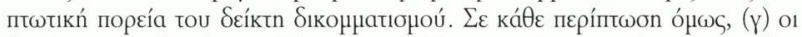

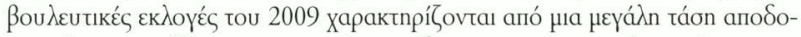

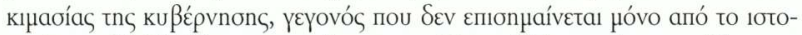

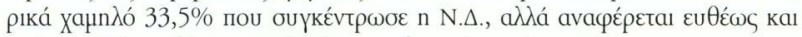

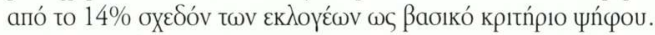

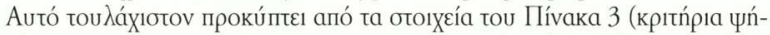

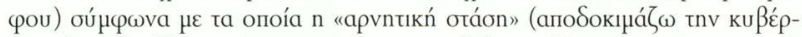

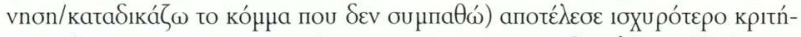

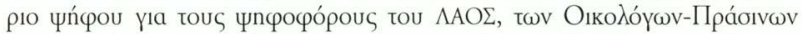

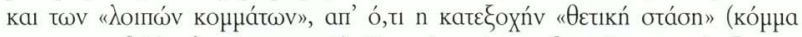

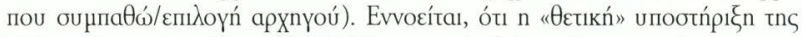

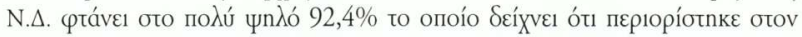

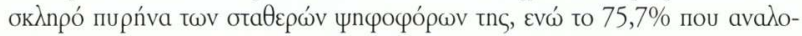

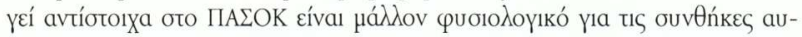

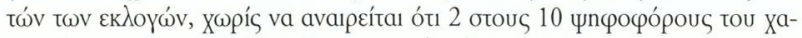

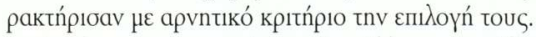

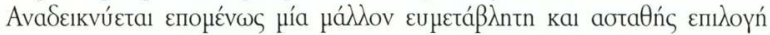

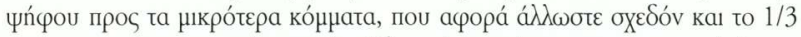

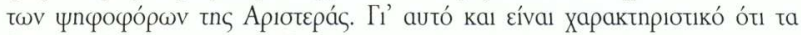

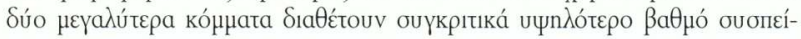




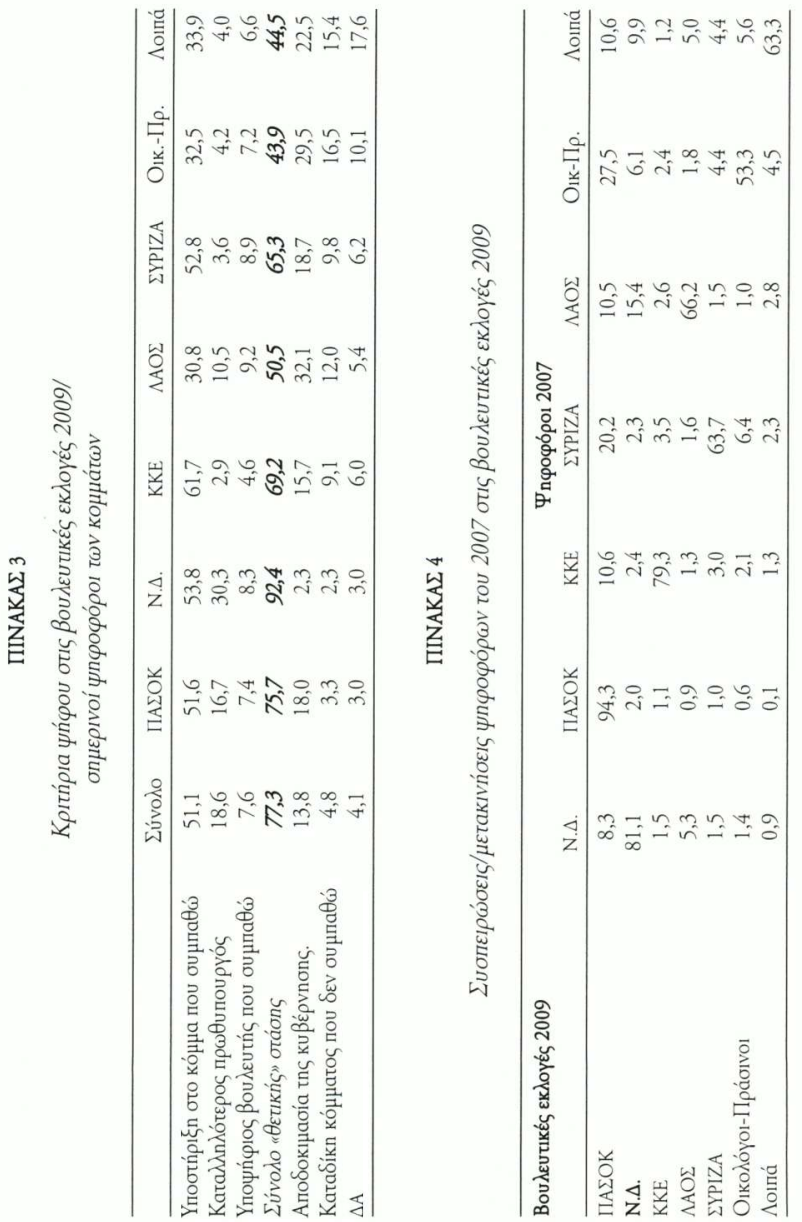




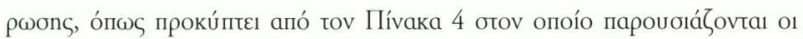

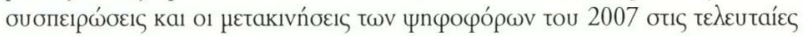

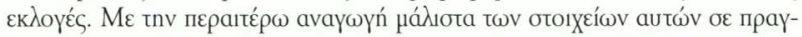

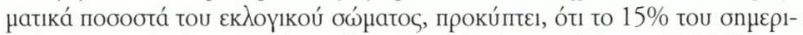

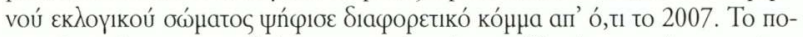

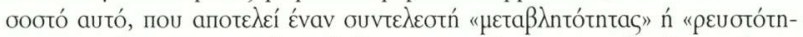

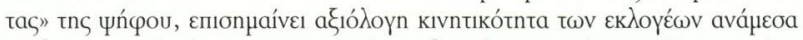

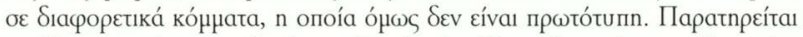
ов ó

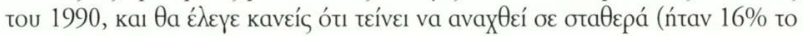
2007 кa1 $14 \%$ to 2004$).$

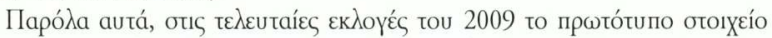

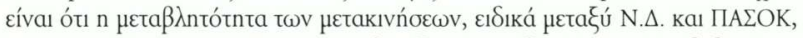

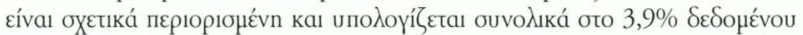

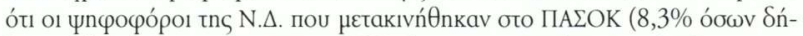

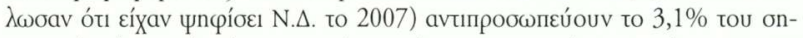

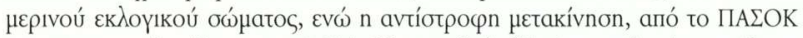

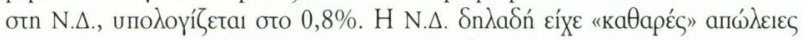

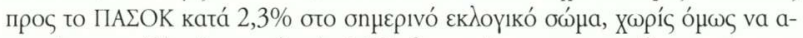

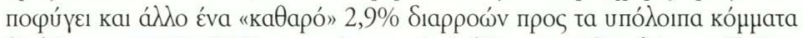

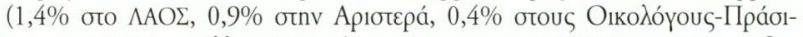

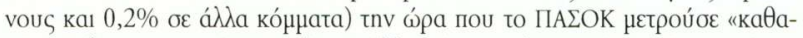

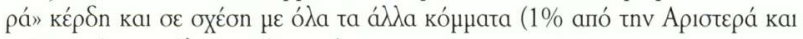

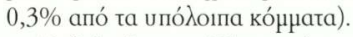

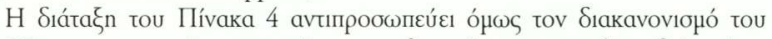

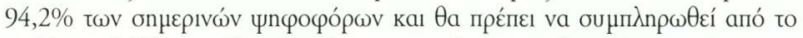

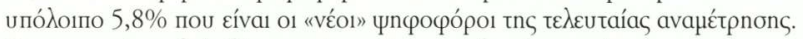

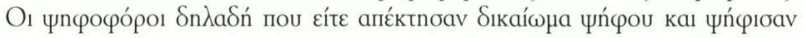

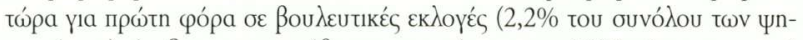

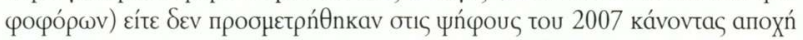

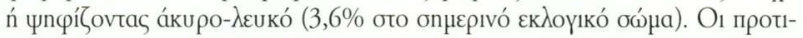

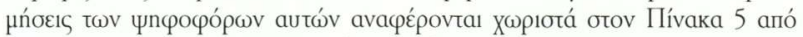

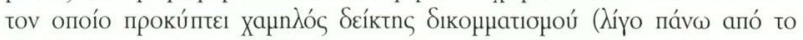

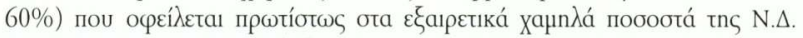

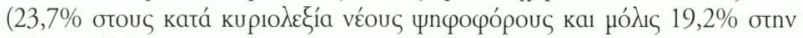

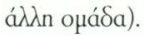

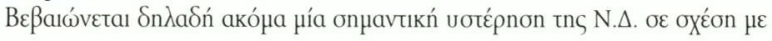




\section{ПINAKA 5}

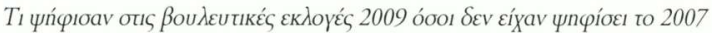

\begin{tabular}{|c|c|c|}
\hline & 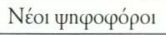 & 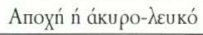 \\
\hline ПАЕОК & 41,6 & 41,4 \\
\hline N. $\Delta$. & 23,7 & 19,2 \\
\hline KKE & 9,7 & 7,8 \\
\hline$\Lambda A O \Sigma$ & 9,1 & 6,9 \\
\hline EYPIZA & 5,7 & 5,5 \\
\hline Оıкоһóүoı-Пра́оıvоı & 4,7 & 8,3 \\
\hline 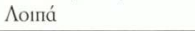 & 5,5 & 10,9 \\
\hline
\end{tabular}

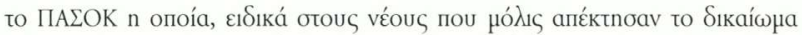

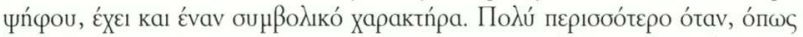

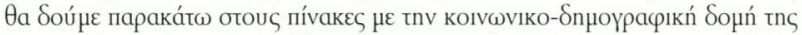
чń

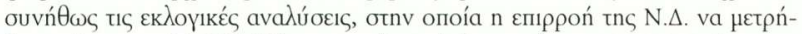

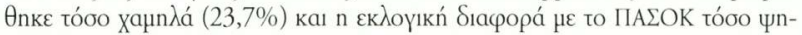

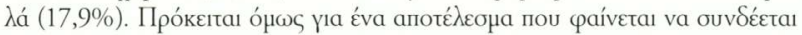

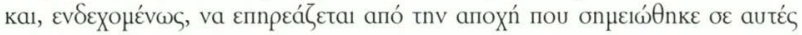

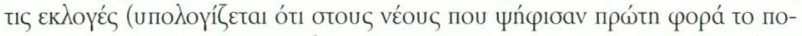

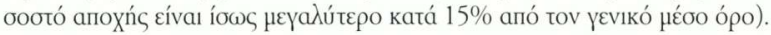

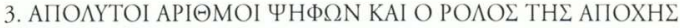

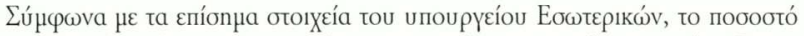

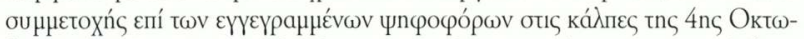

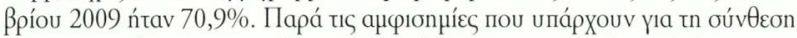

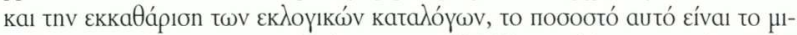

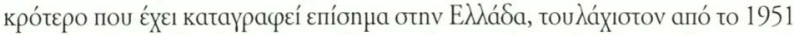

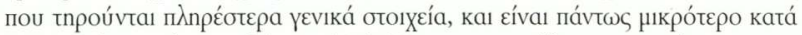

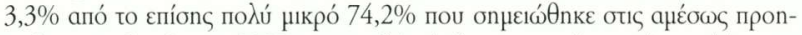

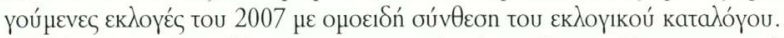

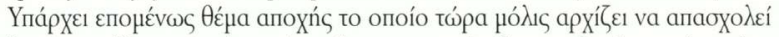

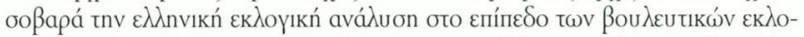

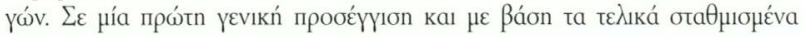




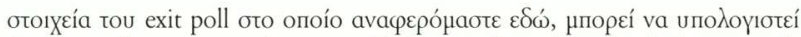

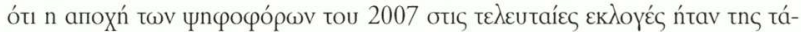

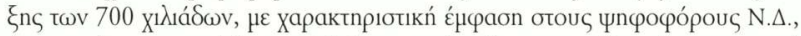

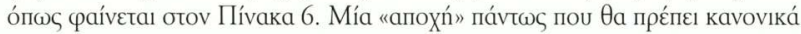

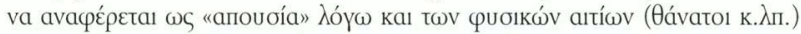

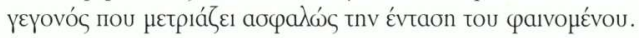

\section{ПINAKA 6}

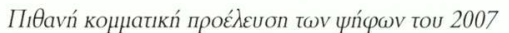

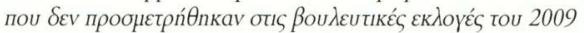

\begin{tabular}{|c|c|c|}
\hline & 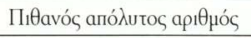 & $\%$ \\
\hline N. $\Delta$. & 425.158 & 60,7 \\
\hline ПАЕОК & 122.481 & 17,5 \\
\hline KKE & 87.892 & 12,5 \\
\hline ¿YPIZA & 45.617 & 6,5 \\
\hline$\Lambda A O \Sigma$ & 10.506 & 1,5 \\
\hline ヘoiná & 8.851 & 1,3 \\
\hline Eúvoגo & 700.505 & 100,0 \\
\hline
\end{tabular}

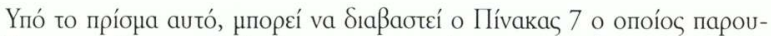

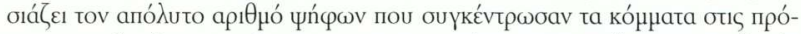

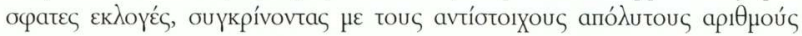

\section{MINAKA 7}

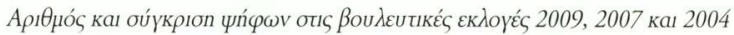

\begin{tabular}{|c|c|c|c|c|}
\hline & B2009 & B2007 & B2004 & B09-B07 \\
\hline ПАЕОК & 3.012 .373 & 2.727 .279 & 3.003 .988 & +285.094 \\
\hline N. $\Delta$ & 2.295 .967 & 2.994 .979 & 3.360 .424 & -699.012 \\
\hline KKE & 517.154 & 583.750 & 436.818 & -66.596 \\
\hline$\Lambda \mathrm{AO} \Sigma$ & 386.152 & 271.809 & 162.492 & +114.343 \\
\hline EYPIZA & 315.627 & 361.101 & 241.714 & -45.474 \\
\hline 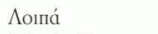 & 331.069 & 220.088 & 202.938 & +110.981 \\
\hline ¿úvoגo/Еүкuра & 6.858 .342 & 7.159 .006 & 7.408 .374 & -300.664 \\
\hline Акшра-Лвика́ & 186.137 & 196.020 & 166.816 & -9.883 \\
\hline In pí̈аvтеs & 7.044 .479 & 7.355 .026 & 7.575 .190 & -310.547 \\
\hline
\end{tabular}




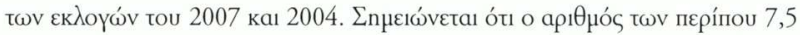

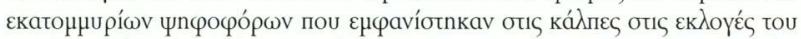

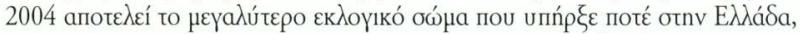

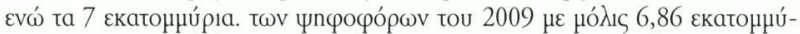

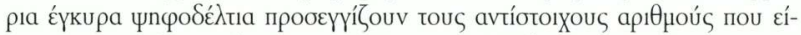

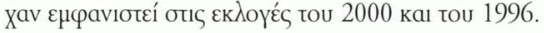

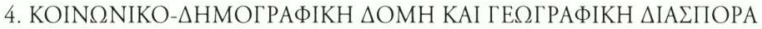 ТНऽ ЧНФОY}

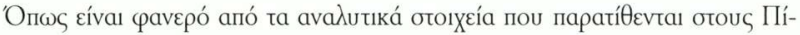

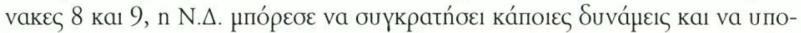

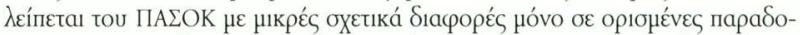

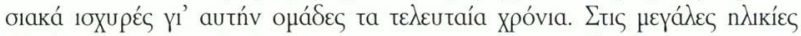

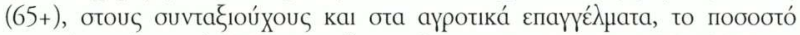

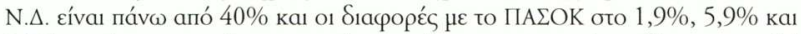

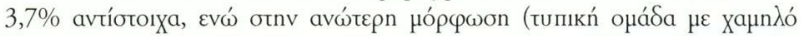

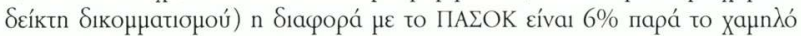

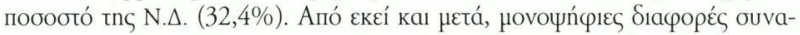

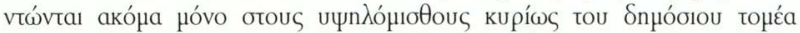

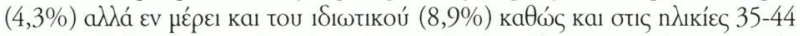

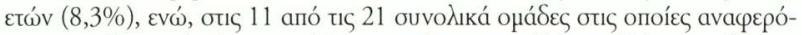

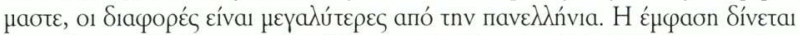

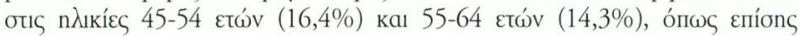

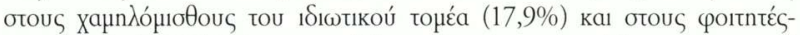

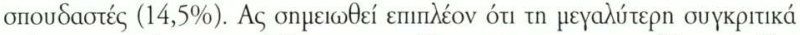

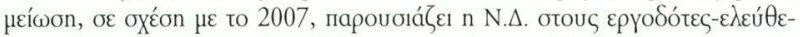

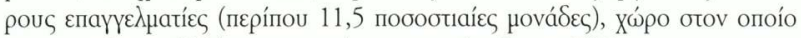

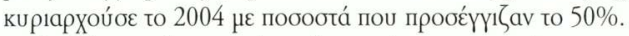

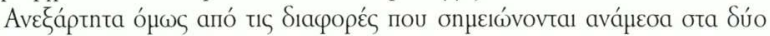

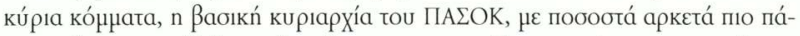

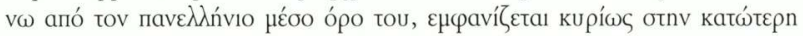

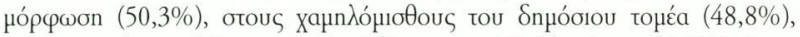

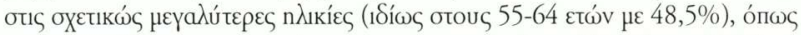

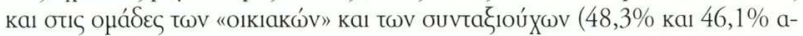

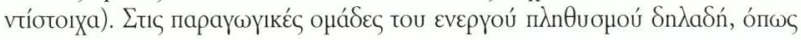




\section{ПINAKA 58}

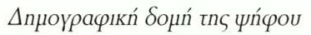

\begin{tabular}{|c|c|c|c|c|c|c|c|c|c|}
\hline & ПАЕОК & N. $\Delta$. & $\begin{array}{l}\text { ПА乏OK } \\
-N . \Delta .\end{array}$ & 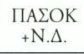 & KKE & $\triangle A O \Sigma$ & ¿YPIZA & OIK. & Moıná \\
\hline \multicolumn{10}{|l|}{$\Phi Y \Lambda \Lambda O$} \\
\hline 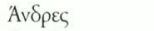 & 43,5 & 33,0 & 10,5 & 76,5 & 7,6 & 6,9 & 3,9 & 2,3 & 2,7 \\
\hline Tuvaíkes & 44,4 & 34,0 & 10,4 & 78,4 & 7,4 & 4,1 & 5,4 & 2,7 & 2,1 \\
\hline \multicolumn{10}{|l|}{ HAIKIA } \\
\hline 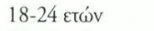 & 42,8 & 29,3 & 13,5 & 72,1 & 7,4 & 5,0 & 6,3 & 3,7 & 5,5 \\
\hline 25-34 عтஸ́) & 40,8 & 30,8 & 10,0 & 71,6 & 6,9 & 7,7 & 5,0 & 4,0 & 4,8 \\
\hline 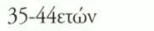 & 41,4 & 33,1 & 8,3 & 74,5 & 6,4 & 7,4 & 5,6 & 3,8 & 2,3 \\
\hline 45-54 et心́) & 45,4 & 29,0 & 16,4 & 74,4 & 10,1 & 5,2 & 5,8 & 2,6 & 1,8 \\
\hline 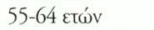 & 48,5 & 34,2 & 14,3 & 82,7 & 7,3 & 4,3 & 3,7 & 1,2 & 0,9 \\
\hline 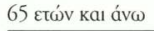 & 44,6 & 42,7 & 1,9 & 87,3 & 6,9 & 3,4 & 1,8 & 0,1 & 0,5 \\
\hline
\end{tabular}

MINAKA 9

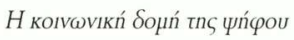

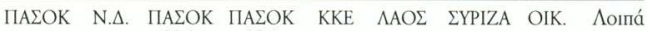
-N. $\Delta . \quad+N . \Delta$.

\section{ЕПАГГЕАMA}

Aүpótes

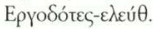

впауу.

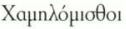

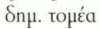

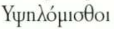

Snн. тоһе́a

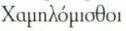

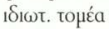

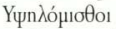

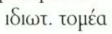

Avepyol

ФoIntés

Oıкıакá

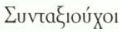

МОРФऽЕН

Katẃtepn

Méon

Avต́tยрn

$\begin{array}{lllllllll}44,3 & 40,6 & 3,7 & 84,9 & 7,0 & 5,0 & 1,8 & 0,7 & 0,7\end{array}$

$\begin{array}{lllllllll}42,5 & 30,0 & 12,5 & 72,5 & 7,3 & 7,3 & 5,3 & 3,9 & 3,7\end{array}$

$\begin{array}{lllllllll}48,8 & 36,1 & 12,7 & 84,9 & 4,4 & 6,3 & 2,7 & 0,8 & 1,0\end{array}$

$\begin{array}{lllllllll}37,6 & 33,3 & 4,3 & 70,9 & 8,5 & 5,7 & 10,0 & 2,9 & 2,1\end{array}$

$\begin{array}{lllllllll}45,1 & 27,2 & 17,9 & 72,3 & 10,0 & 6,9 & 4,7 & 3,1 & 3,1\end{array}$

$\begin{array}{lllllllll}38,3 & 29,4 & 8,9 & 67,7 & 8,7 & 6,7 & 7,9 & 5,2 & 3,9\end{array}$

$\begin{array}{lllllllll}43,3 & 29,6 & 13,7 & 72,9 & 9,9 & 6,7 & 5,6 & 3,2 & 1,7\end{array}$

$\begin{array}{lllllllll}40,9 & 26,4 & 14,5 & 67,3 & 7,3 & 5,4 & 8,8 & 3,8 & 7,3\end{array}$

$\begin{array}{lllllllll}48,3 & 37,9 & 10,4 & 86,2 & 5,9 & 3,4 & 1,7 & 1,8 & 1,1\end{array}$

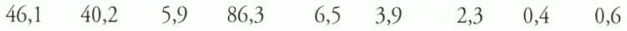

$\begin{array}{lllllllll}50,3 & 37,1 & 13,2 & 87,4 & 6,9 & 3,1 & 1,2 & 0,5 & 0,8\end{array}$

$\begin{array}{lllllllll}44,1 & 32,3 & 11,8 & 76,4 & 8,2 & 6,4 & 4,0 & 2,4 & 2,6\end{array}$

\begin{tabular}{lllllllll}
38,4 & 32,4 & 6,0 & 70,8 & 7,0 & 6,2 & 8,4 & 4,3 & 3,3 \\
\hline
\end{tabular}




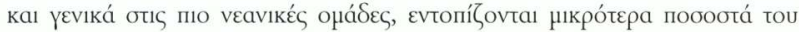

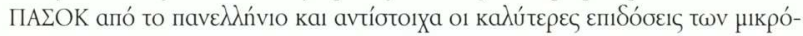

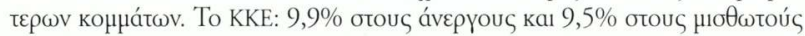

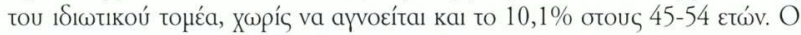

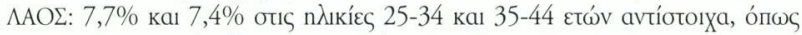

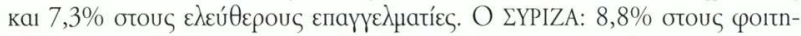

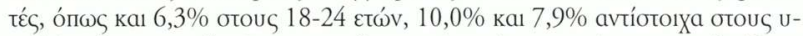

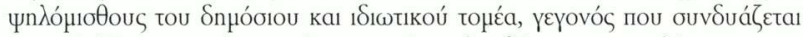

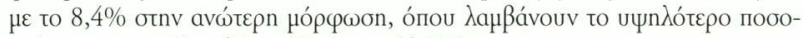

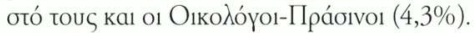

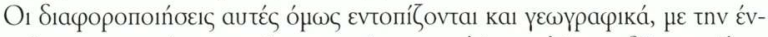

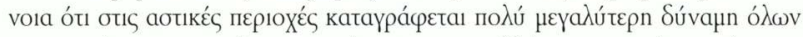

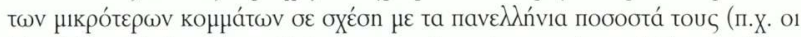

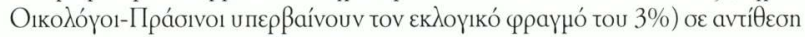
$\mu \varepsilon$ tıৎ̧ Uпо́

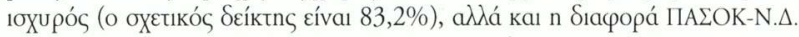

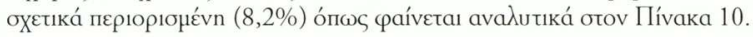

\section{ПINAKA $\Sigma 10$}

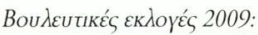

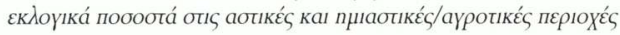

\begin{tabular}{|c|c|c|}
\hline & Aơikés & 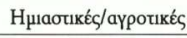 \\
\hline ПАЕОК & 42,2 & 45,7 \\
\hline N. $\Delta$. & 29,7 & 37,5 \\
\hline KKE & 8,8 & 6,1 \\
\hline$\triangle A O \Sigma$ & 6,5 & 4,7 \\
\hline ¿YPIZA & 5,9 & 3,2 \\
\hline Oıкоגóүо1-Пра́бıvol & 3,4 & 1,6 \\
\hline Moirá & 3,5 & 1,3 \\
\hline 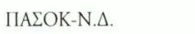 & 12,5 & 8,2 \\
\hline ПАЕОK+N.A. & 71,9 & 83,2 \\
\hline
\end{tabular}

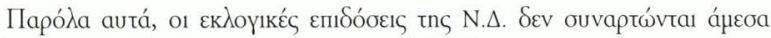

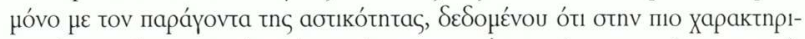

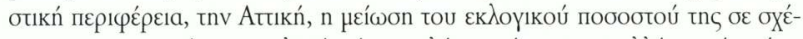

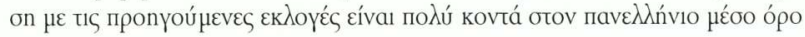




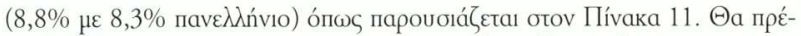

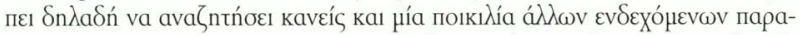

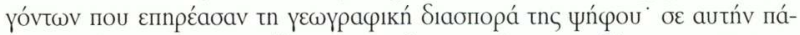

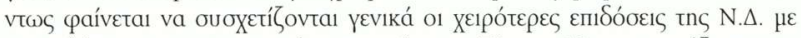

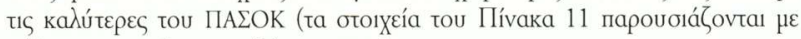

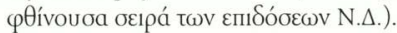

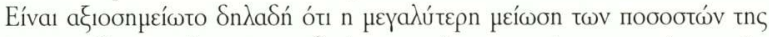

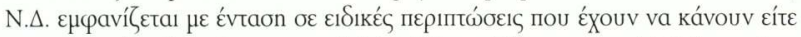

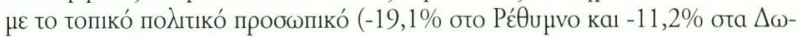

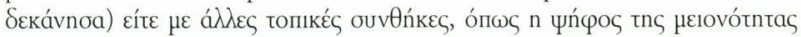

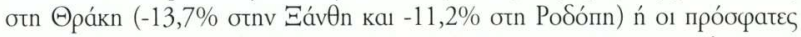

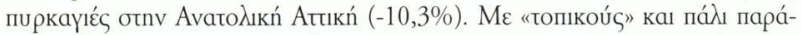

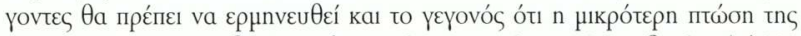

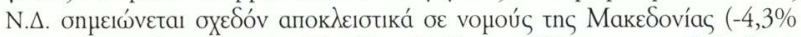

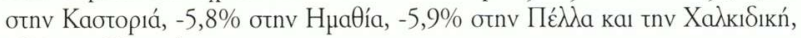

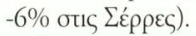

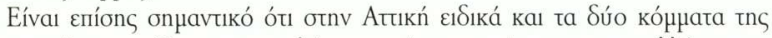

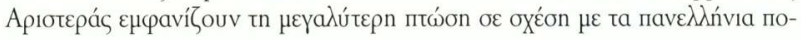

\section{MINAKA $\Sigma 11$}

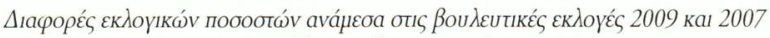

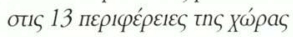

\begin{tabular}{|c|c|c|c|c|c|c|}
\hline & N. $\Delta$. & ПАЕОК & KKE & EYPIZA & $\triangle A O \Sigma$ & Moiná \\
\hline$\Sigma Y N O \Lambda O X \Omega P A \Sigma$ & $-8,3$ & $+5,8$ & $-0,6$ & $-0,4$ & $+1,8$ & $+1,8$ \\
\hline NOTIO AIIAIO & $-10,3$ & $+7,8$ & $-0,3$ & $-0,5$ & $+1,3$ & $+2,1$ \\
\hline КРНTH & $-9,9$ & $+7,4$ & $-0,3$ & $-0,6$ & $+1,6$ & $+1,9$ \\
\hline AN. MAKE $\triangle-\Theta P A K H$ & $-9,4$ & $+5,7$ & $-0,5$ & $+0,9$ & $+2,3$ & $+1,1$ \\
\hline$\Sigma T . E \Lambda \Lambda A \triangle A$ & $-9,3$ & $+6,5$ & $-0,4$ & $-0,4$ & $+2,1$ & $+1,5$ \\
\hline ATTIKH & $-8,8$ & $+6,3$ & $-1,2$ & $-1,3$ & $+2,2$ & $+2,8$ \\
\hline ВOPEIO АIГАIO & $-8,7$ & $+5,8$ & $-0,2$ & $-0,1$ & $+1,6$ & $+1,6$ \\
\hline 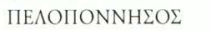 & $-8,4$ & $+6,1$ & $-0,3$ & $-0,5$ & $+1,8$ & $+1,3$ \\
\hline$\triangle$ YTIKH E $\Lambda$ A $\triangle \mathrm{A}$ & $-8,3$ & $+6,8$ & $-0,3$ & $-0,5$ & $+1,1$ & $+1,2$ \\
\hline HIIEIPOE & $-7,8$ & $+5,4$ & $-0,3$ & $+0,3$ & $+1,1$ & $+1,2$ \\
\hline$\Theta E \Sigma \Sigma A \wedge I A$ & $-7,7$ & $+4,7$ & $-0,4$ & $+0,1$ & $+2,0$ & $+1,3$ \\
\hline IONIO & $-7,0$ & $+5,8$ & $-1,5$ & $+0,1$ & $+1,4$ & $+1,3$ \\
\hline$\triangle Y T$. MAKE $\triangle O N I A$ & $-6,7$ & $+4,4$ & $-0,1$ & $+0,2$ & $+1,3$ & $+0,9$ \\
\hline KENTP. MAKE $\triangle O N I A$ & $-6,5$ & $+4,0$ & $-0,5$ & $-0,1$ & $+1,8$ & $+1,3$ \\
\hline
\end{tabular}




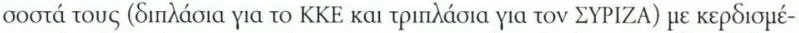

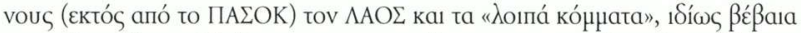

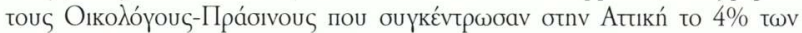

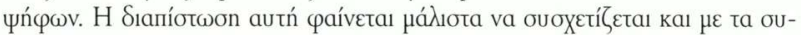

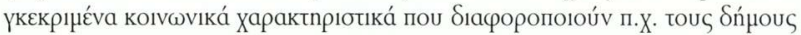

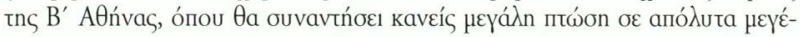

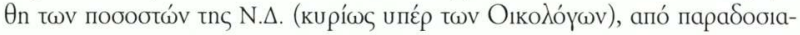

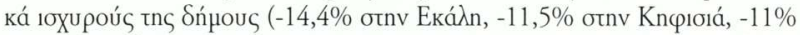

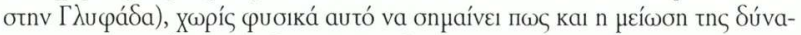

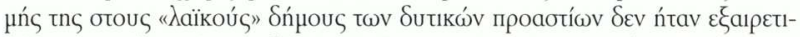

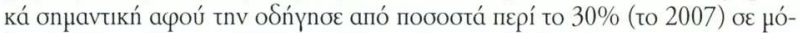

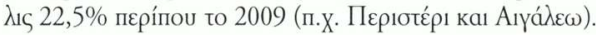

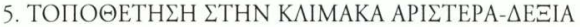

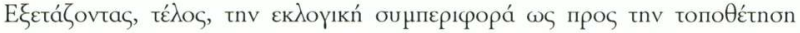

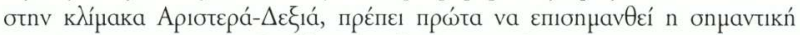

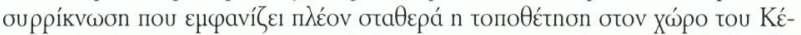

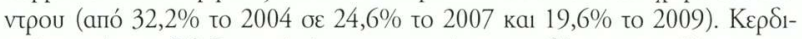
б

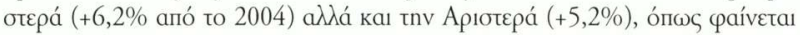

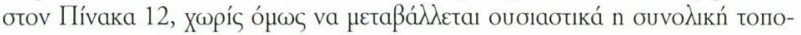

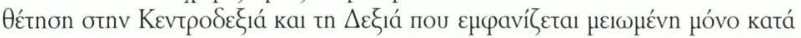

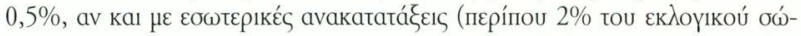

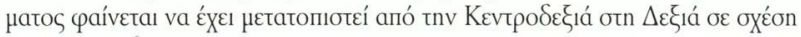
$\mu \varepsilon$ то 2004). ${ }^{6}$

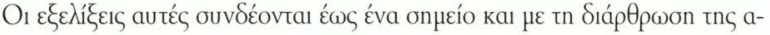

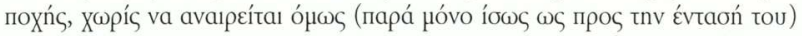

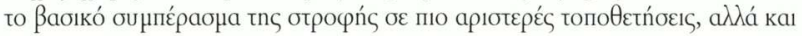

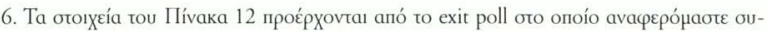

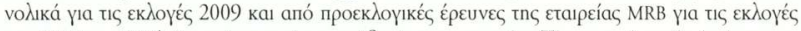

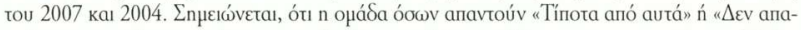

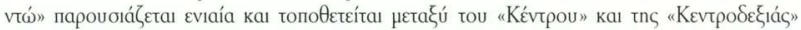

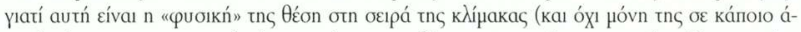

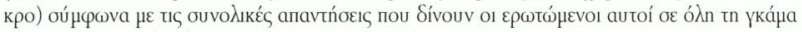

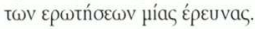




\section{ПINAKA 12}

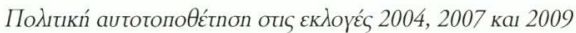

\begin{tabular}{|c|c|c|c|c|c|}
\hline & 2004 & 2007 & 2009 & 2007-2004 & 2009-2007 \\
\hline Арıбтера́ & 7,4 & 9,3 & 12,6 & $+1,9$ & $+3,3$ \\
\hline 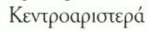 & 12,5 & 14,0 & 18,7 & $+1,5$ & $+4,7$ \\
\hline Kévтро & 32,2 & 24,6 & 19,6 & $-7,6$ & $-5,0$ \\
\hline Tínota/ $\Delta \mathrm{A}$ & 13,3 & 18,1 & 15,0 & $+4,8$ & $-3,1$ \\
\hline 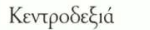 & 14,6 & 11,6 & 12,2 & $-3,0$ & $+0,6$ \\
\hline$\Delta \varepsilon \xi \xi a ́$ & 20,0 & 22,4 & 21,9 & $+2,4$ & $-0,5$ \\
\hline
\end{tabular}

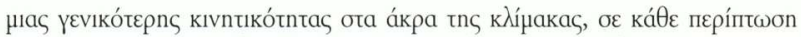

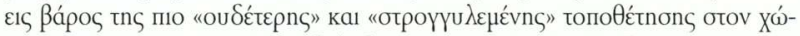

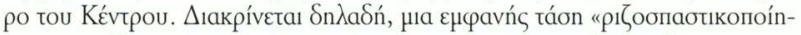

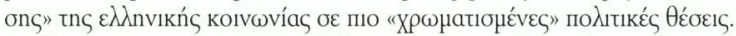

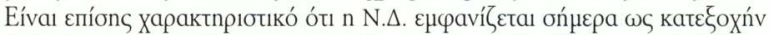

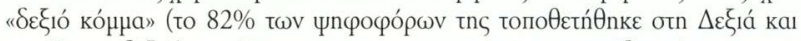

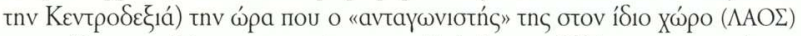

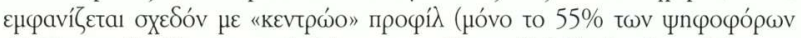

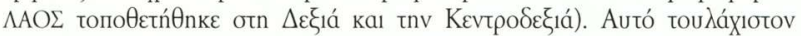

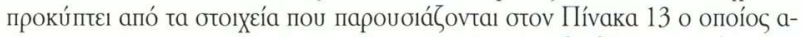

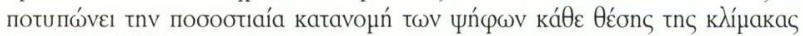

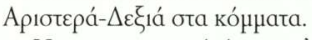

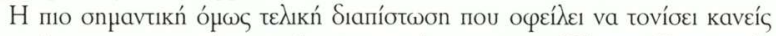

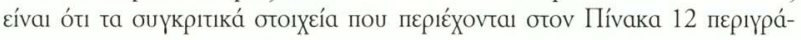

\section{MINAKA $\Sigma 13$}

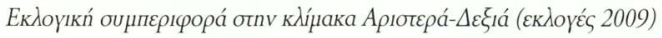

\begin{tabular}{|c|c|c|c|c|c|c|}
\hline & A & $\mathrm{K} / \mathrm{A}$ & $\mathrm{K}$ & Тínота- $\Delta \mathrm{A}$ & $K / \Delta$ & $\Delta$ \\
\hline ПАЕOK & 30,4 & 83,2 & 82,0 & 40,0 & 13,2 & 3,7 \\
\hline N. $\Delta$. & 0,6 & 1,8 & 10,1 & 24,2 & 74,0 & 84,3 \\
\hline KKE & 43,0 & 4,3 & 1,2 & 5,2 & 0,9 & 0,6 \\
\hline$\triangle A O \Sigma$ & 0,9 & 0,7 & 2,6 & 11,7 & 9,2 & 9,0 \\
\hline ¿YPIZA & 17,5 & 7,1 & 0,9 & 4,8 & 0,6 & 0,4 \\
\hline Оıкодо́үов & 2,3 & 1,8 & 2,0 & 7,8 & 1,4 & 0,7 \\
\hline 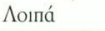 & 5,2 & 1,1 & 1,2 & 6,3 & 0,7 & 1,3 \\
\hline
\end{tabular}




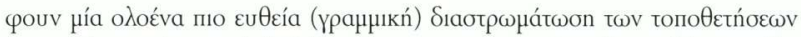

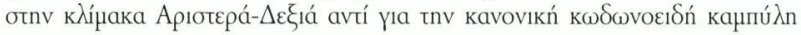

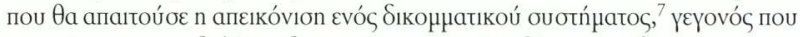

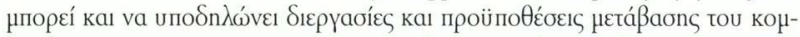

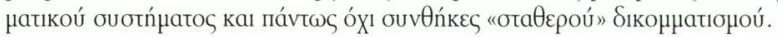

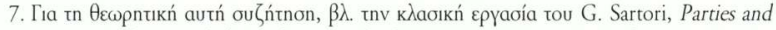
party systems. A framework for analysis, Cambridge University Press, 1976, 10íw o. 188-192,

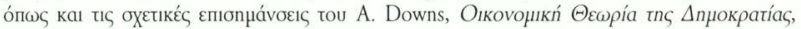

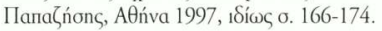

